Environnement Urbain

Urban Environment

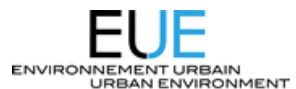

\title{
L'appropriation de sites et paysages urbains requalifiés. Regard sur le Vieux-Port de Chicoutimi
}

\author{
Martin Simard, Marie-José Fortin et Marie-Hélène Tremblay
}

Volume 3, 2009

Urbanisme et développement durable

Urban Planning and Sustainable Development

URI : https://id.erudit.org/iderudit/044599ar

DOI : https://doi.org/10.7202/044599ar

Aller au sommaire du numéro

\section{Éditeur(s)}

Réseau Villes Régions Monde

ISSN

1916-4645 (numérique)

Découvrir la revue

\section{Citer cet article}

Simard, M., Fortin, M.-J. \& Tremblay, M.-H. (2009). L'appropriation de sites et paysages urbains requalifiés. Regard sur le Vieux-Port de Chicoutimi.

Environnement Urbain / Urban Environment, 3, b-1-b-19.

https://doi.org/10.7202/044599ar
Résumé de l'article

Au cours des années 1980 et 1990, le Québec a vécu une série de requalifications d'espaces portuaires. Ces projets urbains ont donné lieu à de multiples processus d'implantation, plus ou moins participatifs ou partenariaux. Des débats renvoient à la question plus large de la construction du paysage et de l'environnement urbains, ainsi qu'à leur appropriation sociale. Dans ce contexte, il semble pertinent de revenir sur l'une de ces expériences de planification, soit celle du "Vieux-port de Chicoutimi ». Comment et pour qui a été façonné ce paysage urbain requalifié? Quelle est la nature du processus d'appropriation du site par les usagers? Près de dix-sept ans après le réaménagement de cet espace urbain, les résultats d'un sondage montrent la forte appropriation du site, tant du point de vue matériel que sur les plans symbolique et politique. 


\title{
L'APPROPRIATION DE SITES ET PAYSAGES URBAINS REQUALIFIES. REGARD SUR LE VIEUX-PORT DE CHICOUTIMI
}

\author{
Martin SIMARD \\ Marie-José FORTIN \\ Marie-Hélène TREMBLAY
}

\begin{abstract}
I RÉSUMÉ
Au cours des années 1980 et 1990, le Québec a vécu une série de requalifications d'espaces portuaires. Ces projets urbains ont donné lieu à de multiples processus d'implantation, plus ou moins participatifs ou partenariaux. Des débats renvoient à la question plus large de la construction du paysage et de l'environnement urbains, ainsi qu'à leur appropriation sociale. Dans ce contexte, il semble pertinent de revenir sur l'une de ces expériences de planification, soit celle du « Vieux-port de Chicoutimi ». Comment et pour qui a été façonné ce paysage urbain requalifié? Quelle est la nature du processus d'appropriation du site par les usagers? Près de dix-sept ans après le réaménagement de cet espace urbain, les résultats d'un sondage montrent la forte appropriation du site, tant du point de vue matériel que sur les plans symbolique et politique.
\end{abstract}

MOTS-CLÉS - Espaces portuaires, paysage urbain, requalification, appropriation, urbanisme, Chicoutimi

\begin{abstract}
- ABSTRACT
In Québec, a number of cities launched waterfront revitalization initiatives during the 1980's and 1990's. These large projects were carried out through a series of implementation mechanisms that were to some extend participatory in nature. Debates around the planning and design process raised the broader issue of the formation of urban and environmental landscapes and of sense of place. For that reason, the discussion focuses on the implications of the development of the City of Chicoutimi's so-called "Old-Port". How and for whom was this revitalization process implemented? What are the ways in which users of the site define sense of place? More than 17 years after the redevelopment of this area began, survey results show strong support for the site, as much from a strictly material point of view as from a symbolic and political one.
\end{abstract}

KEYWORDS - Waterfront areas, Urban landscape, Revitalization, Sense of place, Urban planning, Chicoutimi

- Coordonnées des auteurs : Martin Simard, Département des sciences humaines et CRDT, Université du Québec à Chicoutimi, courriel : MartinG_Simard@uqac.ca; Marie-José Fortin, Chaire de développement régional et territorial, Université du Québec à Rimouski; Marie-Hélène Tremblay, Département des sciences humaines, Université du Québec à Chicoutimi. 


\section{INTRODUCTION'}

Au cours des années 1980 et 1990, le Québec a vécu une série de grands programmes urbanistiques visant à requalifier des espaces portuaires, notamment à Montréal, à Québec, à Trois-Rivières et à Chicoutimi. Divers projets d'aménagement ont ainsi été mis en place, ciblant généralement les fonctions récréative et touristique pour ces territoires centraux situés en bordure de plans d'eau importants. Ces démarches s'inscrivent dans un contexte culturel marqué par une plus grande sensibilité face à la qualité de vie, à l'environnement et au patrimoine (Bonin, 2008). De plus, elles s'insèrent dans une tendance relativement nouvelle, qualifiée d'urbanisme festif, laquelle tendance aspire à revaloriser symboliquement les centres-villes par des aménagements permanents ou temporaires (Zuchin, 1995 ; Pradel, 2007). Sur le plan pratique, ces interventions s'expliquent par une conjoncture caractérisée par la relocalisation des activités industrialo-portuaires vers la périphérie urbaine et par la politique fédérale de cession des infrastructures portuaires aux municipalités locales.

Les projets québécois de requalification d'espaces portuaires ont donné lieu à de multiples processus d'implantation, plus ou moins participatifs ou partenariaux (Courcier, 2008; Larochelle et Piché, 1990 ; Marsan, 1991). On y remarque des débats ou des conflits opposant les élites locales à des groupes de citoyens, au sujet des usages à favoriser ou de la forme des aménagements (Tremblay, 2004 ; Villeneuve et al., 2005). De plus, les projets de réaménagement ont pu contenir des espaces verts, minéralisés ou construits dans des proportions différenciées. Par ailleurs, la « popularité » de ces sites requalifiés varie d'un endroit à l'autre. Finalement, la nature des règles de fonctionnement, de même que la pérennité du concept originel d'aménagement, sont également diversifiées.

Les débats entourant le réaménagement d'anciennes zones portuaires renvoient à la question plus large de la construction du paysage et de l'environnement urbains, ainsi que de l'appropriation sociale de ceux-ci. En effet, de tels projets d'urbanisme constituent des temps forts dans l'histoire des villes, en particulier celle des villes moyennes. II s'agit souvent d'occasions pour, sinon les redessiner, du

\footnotetext{
I Cette recherche a été financée par le programme «nouvelle initiative » du Centre de recherche sur le développement territorial (CRDT) et par la Chaire de recherche du Canada en développement régional et territorial (UQAR). De plus, les auteurs aimeraient remercier Madame Johanne Boisjoly pour ses précieux commentaires sur les aspects méthodologiques de l'analyse.
}

moins tenter de donner un second souffle à leur centre plus ancien, généralement délaissé au profit d'espaces suburbains. Mais comment ont été façonnés ces nouveaux paysages urbains? Et pour qui? En d'autres termes, situer ces projets urbanistiques dans une perspective de paysage oblige à poser la question de l'appropriation des grands espaces publics comme les espaces portuaires requalifiés, et cela, autant en termes matériels qu'en termes symboliques.

Comme le suggèrent divers chercheurs et certaines politiques publiques (Conseil de l'Europe, 2000), le paysage n'est pas tant la partie visible des formes matérielles et l'effet de composition que la façon de percevoir ces éléments et de leur attribuer une signification. Dans cette perspective, comme le formule Augustin, «la construction et l'aménagement de sites publics participent à la fabrication d'identités communes puisqu'ils s'inscrivent dans l'espace vécu et quotidien des groupes et des habitants » (2000: II). L'enjeu de l'appropriation, matérielle et symbolique, est donc au cœur des pratiques et des recherches en architecture de paysage, en urbanisme et en aménagement du territoire. En effet, la finalité première de tout aménagement n'est-elle pas que celui-ci soit utilisé et apprécié par les usagers?

Dans ce contexte, il semble pertinent de revenir sur l'une de ces expériences de requalification d'espace portuaire. Inauguré en 1992, le «Vieuxport » de Chicoutimi, dans la ville regroupée de Saguenay, est un exemple intéressant de métamorphose territoriale ayant misé sur le caractère public des aménités. Autant les procédures interpellées que les formes spatiales et paysagères adoptées, dans une situation de ville moyenne, pourraient apporter des enseignements. Nous tenterons de faire un bilan de ce programme d'urbanisme par le biais du concept d'appropriation. Dans cette optique, nous traiterons d'abord de la dimension théorique de ce concept. Nous poursuivrons avec une brève mise au jour de la petite histoire du projet. Par la suite, nous analyserons les résultats d'un sondage tenu en 2008 auprès d'usagers, après avoir abordé les aspects méthodologiques.

\section{L'APPROPRIATION DES ESPACES PUBLICS ET DU PAYSAGE URBAIN}

Le concept d'appropriation est employé depuis plus d'une vingtaine d'années dans les domaines et disciplines qui s'intéressent aux territoires. Curieusement, il existe peu de définitions ou de critères d'analyse de ce phénomène 
sociopsychologique. II ne s'agit pas d'une appropriation au sens d'une prise de possession physique ou légale, mais bien des mécanismes par lesquels les populations apprivoisent un site ou un territoire plus vaste, apprennent à s'y repérer et à s'y sentir à l'aise, pour éventuellement le considérer comme faisant partie de leur identité, d'une certaine manière. Hossard et Jarvin en parlent comme de «l'acte de faire sien par l'attribution d'un sens » (2005: 22). Cette appropriation est une construction sociale qui se réalise dans le temps, à travers la fréquentation des lieux et en fonction de diverses variables, notamment les attributs géoarchitecturaux du paysage et de l'environnement.

Le concept d'appropriation est difficile à traduire dans d'autres langues que le français. En anglais, certains termes s'en rapprochent: sense of place, attachment, territorial marking (Davies et Herbert, 1993). Malgré tout, l'appropriation est un thème qui s'intègre au courant culturaliste en urbanisme, courant très présent chez les Anglo-saxons Jacobs, 196I; Lynch, 1989 ; Mumford, 198I, etc.). Le culturalisme prône une ville plus compacte, conçue d'abord pour les piétons. Dans le monde francophone, l'appropriation a notamment été étudiée en évaluant les effets néfastes de la réglementation par les autorités des usages d'un lieu (Hossard et Jarvin, 2005). Semmoud (2007) a aussi traité de « la réception sociale de l'urbanisme », en mettant en relief la complexité des valeurs et représentations des usagers et les processus de recomposition de celles-ci, occasionnés entre autres par les projets urbains et les opérations de requalification. De manière plus fondamentale, la lisibilité de l'espace selon les groupes sociaux a été examinée. Or, comme l'affirme SerfatyGarzon en revisitant des écrits de Marx, Lefebvre, Chombart de Lauwe et Raymond: « [l]'appropriation est ainsi à la fois une saisie de l'objet et une dynamique d'action sur le monde matériel et social dans une intention de construction du sujet » (2003 : 27).

Dans cette perspective, il est possible de se demander dans quelle mesure les projets d'aménagement réalisés par les professionnels répondent aux besoins et aux goûts des populations. Sachant que les cultures paysagères des premiers et des seconds peuvent différer, comme l'ont souligné diverses recherches, les choix ne sont pas toujours aisés et ils peuvent devenir sources de tensions, voire de conflits (Dubost et Lizet, 1995). Or, des choix sont faits à chacune des phases du processus de planification et ces choix influencent le projet finalement concrétisé. Les phases plus en amont ont retenu l'attention des chercheurs, entre autres, sous l'angle des dynamiques d'acteurs et des processus de participation touchant l'attribution des vocations des sites et des grandes orientations de leur programmation. Quant aux phases plus en aval, relatives aux fonctions, au design et à l'appropriation des sites par les usagers, celles-ci ont été moins investiguées.

Plusieurs tentatives d'opérationnaliser le concept d'appropriation s'inspirent des écrits sur la phénoménologie de l'espace. Par exemple, Simard (2000) a décliné le concept d'appropriation en trois aspects, soit la satisfaction exprimée, la symbolique du lieu et le sentiment de contrôle. La satisfaction serait perceptible dans les avis et opinions sur la qualité du site et sur les bénéfices associés aux aménagements et équipements. La symbolique du lieu est un élément plus intangible, qui se reflète dans l'image du lieu, dans son utilisation effective, ainsi que dans les vertus qu'on lui attribue. Le sentiment de contrôle serait issu de l'ouverture des processus décisionnels aux citoyens et de l'impression que les avis et besoins de la population ont été considérés dans le processus d'implantation et dans la gestion d'un site.

Les recherches réalisées sur la notion de paysage, particulièrement nombreuses depuis une quinzaine d'années, peuvent aussi aider à traduire le concept d'appropriation en des termes opératoires, malgré leur accent sur le milieu rural. Par exemple, Fortin (2005) propose de décomposer l'appropriation autour de trois dimensions complémentaires: la dimension matérielle, la dimension culturelle et la dimension sociopolitique. Chacun des modes d'appropriation peut être associé à un corpus de travaux réalisés en paysage que nous estimons constituer un paradigme. L'appropriation matérielle réfère à l'ensemble des pratiques sociales associées à un site, incluant les usages, les habitudes de fréquentation et l'accessibilité, et se rapprochant plus largement de la notion d'« espace vécu » de Frémont (1976).

En ce qui a trait à l'appropriation culturelle, cette dimension s'inspire du concept de culture paysagère, entendue comme un ensemble de manières de percevoir et de figurer l'espace, qui «posent des formes de perception, manières de voir et manières d'être qui transmettent valeurs et traditions et tissent des liens culturels, tout comme le langage » (Epstein, 1981 : 54). La culture paysagère, comme ensemble de codes et de conventions sociales partagés et assimilés par les membres d'une communauté, participe ainsi à l'établissement de liens sociaux, tout comme à l'exclusion. Elle se cristallise notamment dans des normes touchant l'appréciation esthétique qui peuvent 


\section{EUE • L'appropriation des sites et paysages urbains requalifiés $\bullet b-4$}


Luginbühl, 1995). L'appropriation culturelle réfère donc aux processus de perceptions et de qualification des lieux: par le biais de l'expérience sensorielle, touchant l'esthétique des formes, elle renvoie au rapport au lieu et à l'environnement naturel, de même qu'aux significations qui en découlent.

Enfin, l'appropriation sociopolitique est liée au pouvoir du sujet dans l'espace public. D'une part, elle rejoint la notion de territorialité, c'est-à-dire de la maîtrise de l'espace par un ou plusieurs groupes d'acteurs. Par exemple, l'aménagement a-t-il été conçu pour répondre aux besoins des uns plus qu'à ceux des autres? D'autre part, ce mode d'appropriation concerne le rapport à l'Autre: est-il possible de s'identifier à l'espace public et d'y « vivre-ensemble »? Dans ce processus de questionnement, Raffestin estime que le paysage constituerait un « système de communication » dont le rôle serait de transmettre « un message sur l'extériorité et l'altérité » (1977: 126). En ce sens, la représentation du paysage et l'image du site portées par des individus et des groupes pourraient pourraient indiquer la présence d'une appropriation sur un site comme la zone portuaire de Chicoutimi.
Ainsi, les grandes opérations de requalification d'espaces portuaires représentent des moments importants pour dessiner la ville et, idéalement, essayer de faire en sorte que celle-ci réponde aux divers besoins de ses citoyens. Les étapes de la planification de ces grands sites urbains, en amont, ont été plus étudiées que celles réalisées en aval. Notre étude vise à apporter une connaissance au sujet de l'appropriation de l'espace urbain, en s'intéressant aux usagers qui fréquentent un site situé dans une ville moyenne du Québec, soit le «Vieux-Port» de Chicoutimi. Est-ce que les choix faits par les aménagistes répondent aux aspirations des visiteurs? Pour répondre à cette question, nous tentons de saisir de quelles façons les visiteurs s'approprient ce lieu. Inspirés de travaux précédents, nous distinguons trois formes d'appropriation: matérielle, symbolique et politique (voir Figure I). Nous cherchons donc à comprendre les pratiques et les habitudes de fréquentation du site, la façon dont les usagers qualifient les choix faits en matière d'esthétique formelle, s'ils estiment que le site participe à leur qualité de vie, qu'il représente bien l'image de Chicoutimi, etc. Avant de décrire plus avant la méthodologie utilisée ainsi que les résultats d'enquête, situons brièvement le site et le contexte entourant les choix d'aménagement.

\begin{tabular}{|c|}
\hline Dimension \\
\hline 1) Appropriation matérielle \\
(usages, pratiques et accessibilité, etc.) \\
\hline 2) Appropriation symbolique \\
\hline (appréciation, sens du lieu, sentiment d'être à sa place, etc.) \\
\hline 3) Appropriation politique \\
\hline (sentiment de maîtrise du territoire, rapports à l'altérité, etc.) \\
\hline
\end{tabular}

Fig. I - Les dimensions du concept d'appropriation

\footnotetext{
2 L'idée du modèle peut être associée à celle du style architectural, c'est-àdire à un ensemble de formes et d'éléments matériels créant un effet de composition reconnaissable. En architecture de paysage par exemple, le modèle " pittoresque » a longtemps servi de référence dominante pour penser l'aménagement des grands parcs urbains au début du $20^{\mathrm{e}}$ siècle, dont ceux de Central Park à New York et du Mont-Royal à Montréal.
} 


\section{SITE ET CONTEXTE DU PROJET}

Le centre-ville de Chicoutimi est principalement construit sur des terrasses et des affleurements rocheux constituant le lit majeur de la rivière Saguenay, sur la rive sud du cours d'eau. Ce milieu vallonné et escarpé par endroits regroupe des activités institutionnelles et commerciales dans une concentration importante, malgré le fait que plusieurs entreprises se soient déplacées vers l'axe périphérique du boulevard Talbot depuis les années 1970 (voir Figure 2). À l'intérieur de cet ensemble urbain, le site de la zone portuaire de Chicoutimi est un terrain plat de 17 hectares, dont la majeure partie a été façonnée par des opérations de remblaiement ayant eu lieu en 1929. Cet espace appartenant alors au gouvernement fédéral et géré par le ministère des Travaux publics a été utilisé principalement pour l'entreposage de produits pétroliers et de matériaux en vrac, jusqu'au milieu des années 1980. Les nombreux réservoirs de mazout que l'on y retrouvait ont ainsi profondément affecté le paysage de l'ancienne ville de Chicoutimi et limité l'accès aux berges de la rivière Saguenay, et ce, pendant des décennies (voir Photol).
Vers 1980, la municipalité et des groupes de citoyens prennent conscience des problèmes d'esthétique et des dangers possibles reliés à de telles installations industrielles, situées en plein cœur de la capitale régionale. Au même moment, le contexte favorise la mise en place d'un projet d'envergure au centre-ville de la «Reine-du-Nord». D'une part, le gouvernement canadien annonce la construction d'un autre port, plus aisément accessible, à Grande-Anse, quelques kilomètres en aval sur la rivière. D'autre part, le démantèlement des voies ferrées raccordant le port au réseau régional est à l'ordre du jour. Plusieurs esquisses de projet sont présentées sur la place publiquement au cours des années 1980. Le «VieuxPort » fait l'objet de débats et de propositions à divers moments, notamment lors des campagnes électorales fédérales de 1980, 1984 et 1988.

Pendant près de dix ans, des visions différentes concernant l'avenir du site s'affrontent, comme le résume Tremblay (2004). En 1979, la Société canadienne d'hypothèque et de logement $(\mathrm{SCHL})$ mène une première consultation publique sur le réaménagement de ce grand site. Des visions opposées y sont exprimées. D'un côté, le

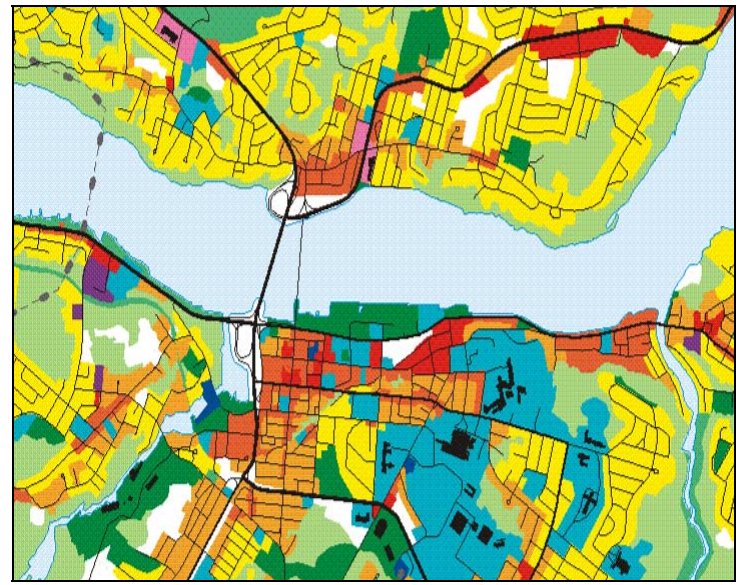

Source : Atlas électronique du Saguenay-Lac-Saint-Jean ville de Chicoutimi

Fig. 2 - Utilisation du sol au centre

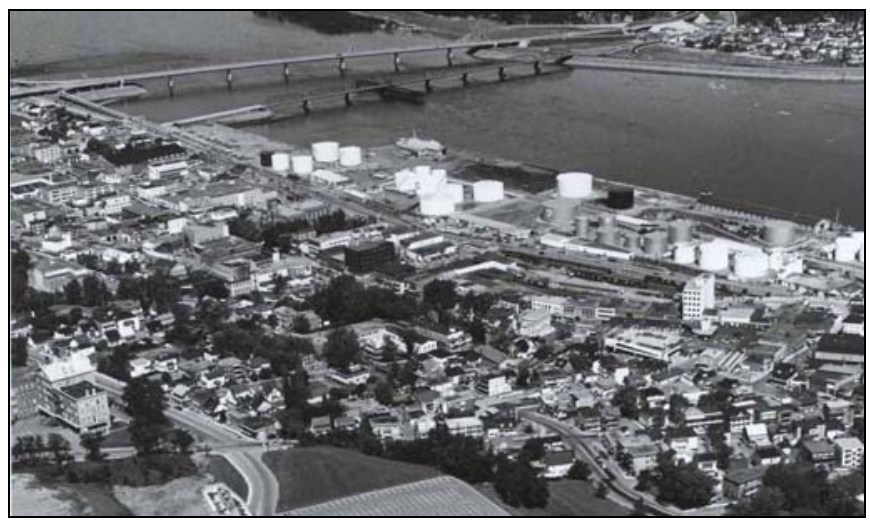

Source : firme Yves-Michel Garant, architecte paysagiste

Photo I - Le centre-ville de Chicoutimi à la fin des années 1970 
gouvernement fédéral propose un programme ambitieux, combinant activités institutionnelles, locatives et récréatives. Plus précisément, le projet est composé « d'un centre de congrès, d'un complexe hôtelier, d'une marina, d'un centre d'achat, d'un édifice administratif et d'un complexe de logements pour la classe moyenne et la classe aisée » (idem: 310). De l'autre, le Comité de l'Environnement de Chicoutimi (CEC) mise sur une conception de l'espace urbain valorisant des habitations locatives destinées à des classes sociales moins fortunées et, surtout, sur la présence d'espaces verts à des fins récréatives et communautaires.

Au cours des années qui suivent, le CEC fait des efforts importants pour mobiliser les acteurs locaux et régionaux autour de sa vision, que l'on peut qualifier de sociale et d'environnementale. En 1983, cet organisme prend la tête d'une coalition rassemblant six groupes communautaires. Par le biais de divers moyens, il travaille aussi à rejoindre l'opinion publique, orchestrant deux pétitions de respectivement 1500 et 5000 noms. À chacune des consultations publiques menées sur le thème du réaménagement de la zone portuaire, le comité présente la conception d'un bon projet d'aménagement. Enfin, il cherche à sensibiliser les élus municipaux et fédéraux à sa cause. Le dossier de la zone portuaire s'inscrit alors dans un cadre plus large concernant l'avenir du centre-ville de Chicoutimi, car le projet est vu comme devant contribuer à la revitalisation du secteur.
À la suite d'une consultation publique tenue en 1984, l'administration municipale présente un nouveau projet d'intervention. Celui-ci rejoint les premières esquisses soumises cinq ans plus tôt par le gouvernement canadien. Cependant, les élections fédérales favorisent un contexte propice à une réorientation du projet et des valeurs sous-jacentes. En effet, le conseiller municipal André Harvey devient alors député du comté Chicoutimi-Le-Fjord sous la bannière du parti Progressiste-Conservateur. De concert avec les autorités locales, il travaillera pour que Travaux publics Canada cède le site à la Ville de Chicoutimi et qu'il finance, du même coup, la décontamination des matériaux meubles qui constituent l'assise du site. D'ailleurs, la faible capacité de support des sols était ressortie comme une contrainte limitant l'implantation de grands bâtiments.

En 1984, les éléments politiques se mettent en place pour amener la réalisation d'un programme d'intervention. L'élaboration du plan directeur du grand site urbain (voir Figure 3) est confiée à la firme Yves-Michel Garant, architecte paysagiste. Au-delà de la controverse sur le transport et l'entreposage en milieu périurbain des sols contaminés, le nouveau projet suscite l'adhésion de l'ensemble des acteurs du développement local. II est le fruit d'une concertation entre les consultants et les représentants de la Ville, les élus et les fonctionnaires. Son inauguration est faite en 1992, soit l'année des célébrations du I50e anniversaire du peuplement euro-canadien dans la région du Saguenay-Lac-Saint-Jean.

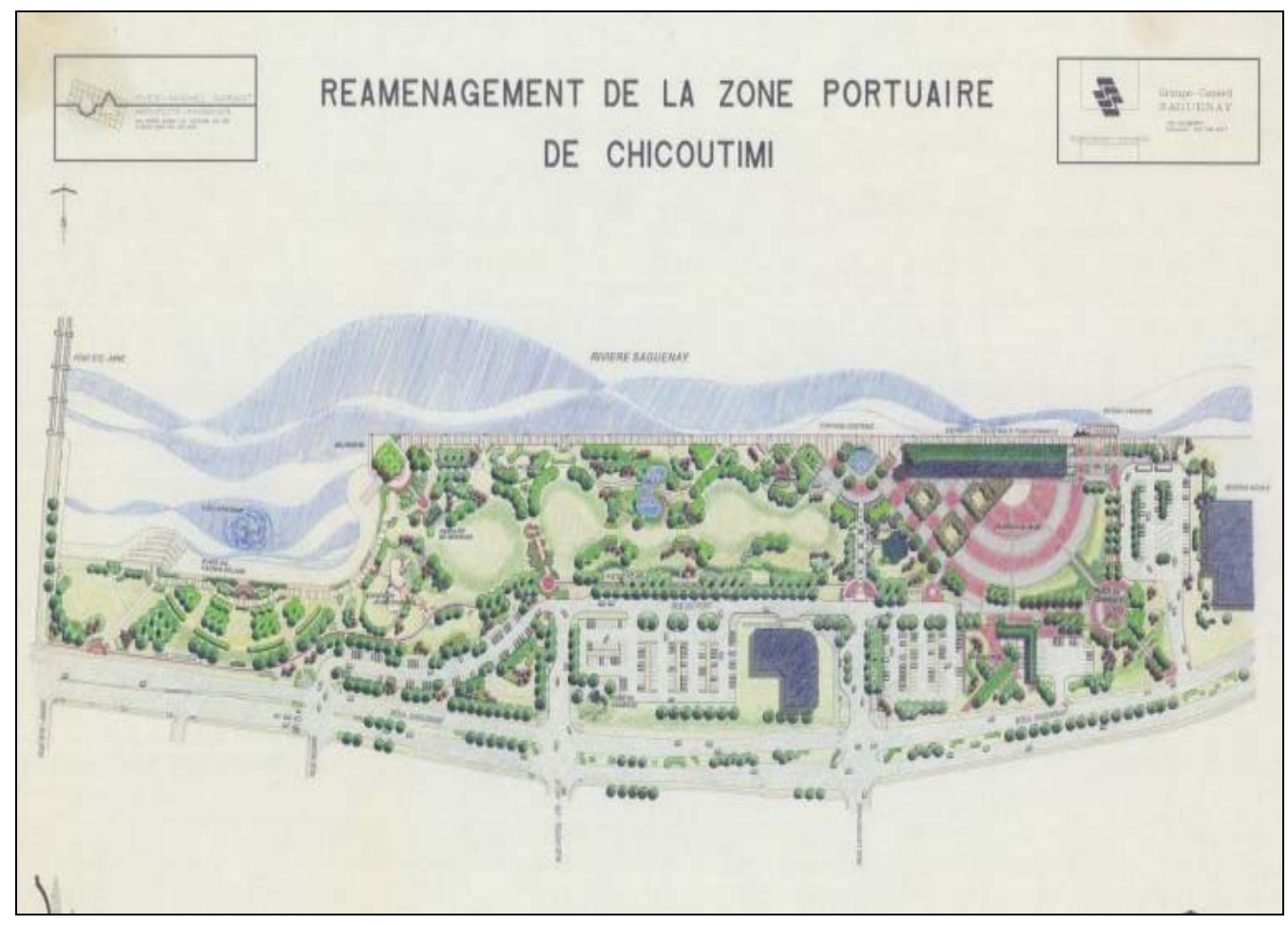

Source : firme Yves-Michel Garant, architecte paysagiste

Fig. 3 - Plan concept du réaménagement de la zone portuaire 


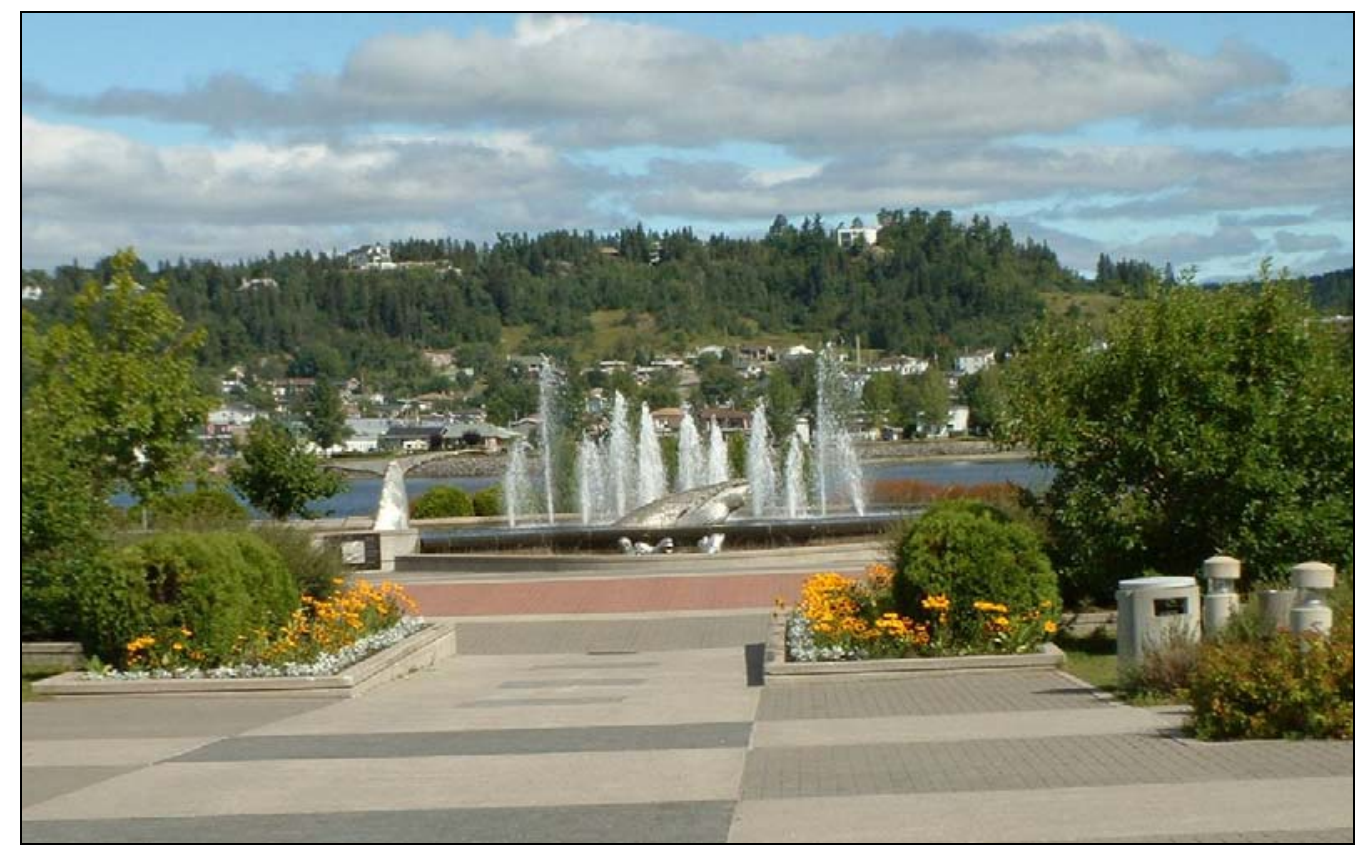

Source : Martin Simard, 2006

Photo 2 - Fontaine située au centre du site

Contrairement au «Vieux-Port » de Québec, le site réaménagé ne comporte pas de logements de luxe de type condominium et il accueille peu de commerces. II s'agit d'un vaste espace paysager accessible à tous et à toutes et relié aux réseaux piétonniers du pont Sainte-Anne et de la promenade du secteur de Rivière-du-Moulin (voir Photo 2). On y retrouve peu de bâtiments, si ce n'est l'ancien hangar et le modeste complexe du marché public des Halles. Toutefois, deux composantes ont affaibli le concept d'aménagement du point de vue des acteurs sociocommunautaires, soit l'implantation d'un édifice administratif fédéral, au centre du site, et celle de la Réserve navale de Chicoutimi, dans sa partie est. Enfin, les activités portuaires ont été restreintes à la présence d'un petit navire de croisières nommé « La Marjolaine » et aux visites occasionnelles de bateaux à faible tirant d'eau, à des fins touristiques.

À première vue, le projet semble exemplaire à plusieurs égards. Le site a gagné plusieurs prix (Boivin, 1993). L'achalandage quotidien y est très fort lors de la période estivale (Tremblay, 1993). Plusieurs activités spéciales s'y déroulent, des activités destinées à des clientèles variées, notamment des spectacles musicaux et de la danse sociale. Cette programmation est développée sous la direction d'un organisme para-municipal de gestion créé à cette fin (Côté, 1993a). Soulignons aussi que les gestes de vandalisme sont rares (Côté, 1993b). Enfin, cet élan de réappropriation populaire d'un site urbain a favorisé un mouvement de revitalisation plus large du centre-ville au cours de la décennie 1990 (voir Photo 3). Ce mouvement, qui a ralenti depuis, intègre l'application du programme «rue principale» sur la rue Racine et l'érection d'un petit centre d'affaires à proximité de l'hôtel de ville (Simard et Maltais, 2004).

Cependant, il semble pertinent de valider cette première lecture par une étude plus approfondie. À cette fin, un sondage a été réalisé. Ce sondage visait à mesurer les formes d'appropriation du site par des usagers, entre autres sous l'angle des usages et habitudes de fréquentation du site, de l'appréciation esthétique des aménagements et de la contribution du site à divers besoins, comme la qualité de vie ou l'image de la ville.

\section{LA MÉTHODOLOGIE DE RECHERCHE}

La population à l'étude, soit les usagers de la zone portuaire de Chicoutimi sur une base annuelle, a été estimée à 200000 personnes par les gestionnaires du site. Ainsi, nous avons choisi de distribuer 385 questionnaires, administrés en face à face par des interviewers, au cours des mois de juillet et août $2008^{3}$. Le questionnaire contenait 31 questions, généralement fermées et à choix de réponses, qui étaient regroupées en trois volets : I) «fréquentation et pratiques reliées

\footnotetext{
3 Pour une telle population, un échantillon de cette taille correspond à une marge d'erreur de plus ou moins $5 \%, 19$ fois sur 20 (Guay, 1991 : 92).
} 


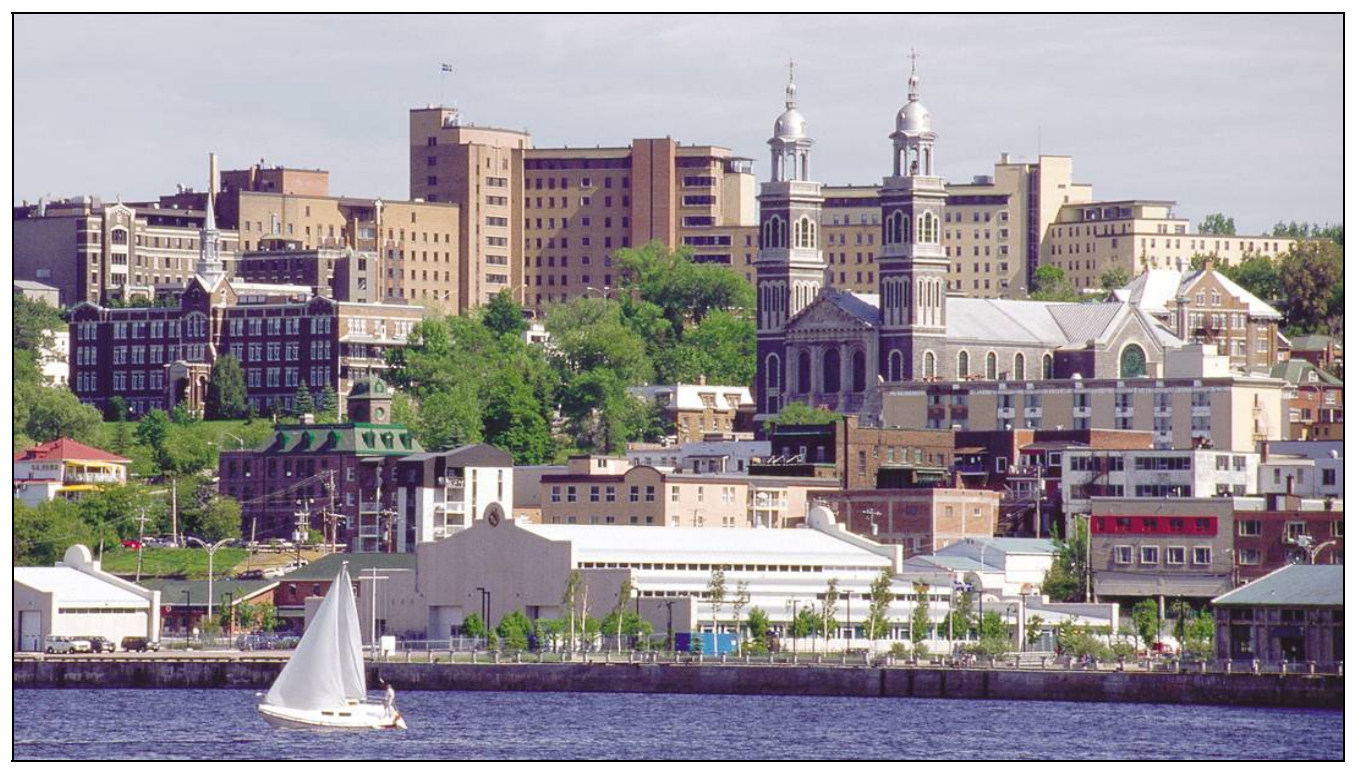

Source : Association des centres-villes de Chicoutimi

Photo 3 - Le centre-ville de Chicoutimi vu de la rive nord du Saguenay

au site », 2) « satisfaction et appréciation du site » ainsi que 3) « identification et profil des répondants ».

Afin que nos résultats soient le plus représentatifs de l'ensemble de la population à l'étude, nous avons choisi de couvrir plusieurs moments de la semaine et du jour, de même que diverses zones à l'intérieur du site. Notre échantillon a donc été divisé en fonction des jours de la semaine et des moments du jour (voir Tableau I). Ensuite, présumant que les gens ont probablement l'habitude de fréquenter un ou des lieux précis sur le site, dépendamment de leur profil, nous nous sommes déplacés sur le site afin de couvrir quatre zones (Promenade ou zone piétonnière; Complexe des Halles et hangar ; Fontaine aux bélugas, étang et jardins; Piste cyclable ou jeux d'enfants). Pour chacune de ces zones, le nombre de questionnaires à administrer a été prédéfini à $25 \%$ de l'échantillon, ce qui a été respecté, à peu de chose près (voir Tableau 2). Notons que le temps maussade lors de la période durant laquelle nous avons effectué notre sondage a soulevé quelques difficultés sur le plan du respect des plages horaires sélectionnées. En ce qui concerne le déroulement des entretiens, le choix des participants se faisait au hasard. Le principe suivant a été adopté : laisser passer trois personnes et interpeller la quatrième personne rencontrée, sauf les jours de faible affluence.

Malgré toutes les précautions prises, la méthode choisie comporte certaines limites. Premièrement, le fait que les répondants aient été des usagers du site plutôt que, plus largement, des citoyens de l'arrondissement, a pu contribuer à augmenter artificiellement les avis et opinions favorables. En effet, la simple fréquentation du lieu est, au départ, une forme d'approbation de l'usage et de la forme du site, car les gens qui n'apprécient pas ce lieu n'auront pas tendance à le visiter. Dans cette perspective, l'enquête ne mesure que l'appropriation des visiteurs interrogés du site. Deuxièmement, nous n'avons pas été en mesure d'évaluer les effets du réaménagement sur l'environnement naturel sur le site de l'ancien port. Une évaluation plus ample, comprenant les processus écologiques, resterait donc à réaliser. De manière pratique, les données ont été traitées à l'aide des logiciels SPSS et Excel. Finalement, notons que nous avons obtenu une certification éthique de la part du Comité d'éthique de la recherche de l'Université du Québec à Chicoutimi.

\section{LES RÉSULTATS DE L'ENQUÊTE}

Les résultats de l'enquête sont présentés en regard des trois dimensions de l'appropriation décrites précédemment, soit les aspects matériels, symboliques et politiques. Mais d'abord, situons le profil des personnes interrogées (voir Tableau 3). L'échantillon est bien divisé entre hommes et femmes. Sur le plan des groupes d'âge et de l'occupation, on remarque un fort contingent de personnes de 50 à 64 ans, ainsi que de retraités. En ce qui concerne les études, les diplômés universitaires sont surreprésentés. Nous avons essayé d'évaluer si l'âge et le niveau de revenu des usagers pouvaient être des variables significatives de l'appropriation du site. À cette fin, des analyses croisées ont été réalisées, dont les résultats sont décrits à l'intérieur de cette section 
EUE • L'appropriation des sites et paysages urbains requalifiés • b-9

Tableau I

La répartition temporelle de l'échantillon

\begin{tabular}{lccc}
\hline & Moment de la journée & Effectifs & $\%$ \\
\hline Avant-midi & 128 & $33,2 \%$ \\
\hline Après-midi & 161 & $41,8 \%$ \\
\hline Soir & 96 & $24,9 \%$ \\
\hline Total & Jour de la semaine & 385 & $100 \%$ \\
\hline & Effectifs & \\
\hline Lundi & 30 & $7,8 \%$ \\
\hline Mardi & 62 & $16,1 \%$ \\
\hline Mercredi & 108 & $28,1 \%$ \\
\hline Jeudi & 80 & $20,8 \%$ \\
\hline Vendredi & 66 & $17,1 \%$ \\
\hline Samedi & 24 & $6,2 \%$ \\
\hline Dimanche & 385 & $15 \%$ \\
\hline Total & & \\
\hline
\end{tabular}

Tableau 2

La répartition géographique de l'échantillon

\begin{tabular}{lcc}
\hline \multicolumn{1}{c}{ Endroit } & Effectifs & $\%$ \\
\hline Promenade ou zone piétonnière & 97 & $25,2 \%$ \\
\hline Complexe des Halles / Hangar & 105 & $27,3 \%$ \\
\hline Fontaine aux bélugas, étang et jardins & 101 & $26,2 \%$ \\
\hline Piste cyclable ou jeux d'enfants & 82 & $21,3 \%$ \\
\hline Total & 385 & $100 \%$ \\
\hline
\end{tabular}


EUE • L'appropriation des sites et paysages urbains requalifiés • b- 10

Tableau 3

Le profil sociodémographique des répondants

\begin{tabular}{|c|c|c|}
\hline Genre & Effectifs & $\%$ \\
\hline Homme & 183 & $47,5 \%$ \\
\hline Femme & 202 & $52,5 \%$ \\
\hline Total & 385 & $100 \%$ \\
\hline Groupe d'âge & Effectifs & $\%$ \\
\hline $15-34$ ans & 92 & $23,9 \%$ \\
\hline $35-49$ ans & 86 & $22,3 \%$ \\
\hline $50-64$ ans & 134 & $34,8 \%$ \\
\hline 65 ans et plus & 73 & $19,0 \%$ \\
\hline Total & 385 & $100 \%$ \\
\hline Occupation & Effectifs & $\%$ \\
\hline En emploi & 187 & $48,6 \%$ \\
\hline Aux études & 38 & $9,9 \%$ \\
\hline Á la maison ou sans emploi & 37 & $9,6 \%$ \\
\hline Retraité & 120 & $31,2 \%$ \\
\hline Autre & 3 & $0,8 \%$ \\
\hline Total & 385 & $100 \%$ \\
\hline Niveau d'études & Effectifs & $\%$ \\
\hline Primaire & 38 & $9,9 \%$ \\
\hline Secondaire & $|4|$ & $36,6 \%$ \\
\hline Collégial & 93 & $24,2 \%$ \\
\hline Universitaire & 109 & $28,3 \%$ \\
\hline Ne sait pas/refus de répondre & 3 & $0,8 \%$ \\
\hline Aucun & $\mathrm{I}$ & $0,3 \%$ \\
\hline Total & 385 & $100 \%$ \\
\hline
\end{tabular}


EUE • L'appropriation des sites et paysages urbains requalifiés • b- I I

Tableau 4

L’appropriation matérielle du site par les répondants

\begin{tabular}{|c|c|c|}
\hline I. Fréquence des visites & Effectifs & $\%$ \\
\hline I fois / semaine & 219 & 56,9 \\
\hline $\mathrm{I}-2$ fois / mois & 53 & 13,8 \\
\hline 2-3 fois / année & 73 & 19,0 \\
\hline lère fois ou moins de 2 fois / année & 40 & 10,4 \\
\hline Total & 385 & 100 \\
\hline 2. Moment préféré pour les visites & Effectifs & $\%$ \\
\hline Sur semaine & 77 & 21,2 \\
\hline Les fins de semaines & 35 & 9,6 \\
\hline Fêtes / événements spéciaux & 38 & 10,4 \\
\hline En tout temps / aucun moment de particulier & 214 & 58,8 \\
\hline Total & 364 & 100 \\
\hline 4. Accompagnement lors des visites & Effectifs & $\%$ \\
\hline Seul & 128 & 35,2 \\
\hline En famille/Avec conjoint & 156 & 42,9 \\
\hline Avec des amis & 71 & 19,5 \\
\hline Autre & 9 & 2,5 \\
\hline Total & 364 & 100 \\
\hline 5. Moyen de transport pour accéder au site & Effectifs & $\%$ \\
\hline Véhicule motorisé & 253 & 69,5 \\
\hline Transport en commun & 8 & 2,2 \\
\hline Vélo, patins ou fauteuil roulant & 17 & 4,7 \\
\hline$\dot{\mathrm{A}}$ pieds & 86 & 23,6 \\
\hline Total & 364 & 100 \\
\hline
\end{tabular}




\section{I Appropriation matérielle de la zone portuaire}

La première dimension traitée concerne l'appropriation matérielle du site. II s'agit de situer les pratiques de fréquentation et d'usages reliées au site. Six questions ont été abordées en ce sens. Tout d'abord, les personnes ont été interrogées sur la fréquence et le moment de leurs visites sur le site de la zone portuaire (Q. I - voir Tableau 4). II apparaît que la clientèle rencontrée est principalement formée de visiteurs réguliers, car $56,9 \%$ des répondants ont indiqué venir une fois ou plus par semaine (au cours de la période d'été). De plus, une proportion semblable de répondants $(58,5 \%)$ ont affirmé fréquenter le site à divers moments de la semaine ou du jour, sans préférence particulière (Q. 2). En outre, la majorité des visiteurs questionnés disent se déplacer en groupes (avec leur conjoint ou leur famille - 42,9\%, avec des amis - 19,5\%) plutôt que de venir seuls (35,2 \% - Q. 4). Par ailleurs, les répondants viennent sur la zone portuaire au moyen de leur voiture ou d'une motocyclette dans une proportion de $69,5 \%$ alors que $23,6 \%$ des visiteurs arrivent sur le site à pieds (Q.5). Finalement, une forte majorité de répondants considère que «le site est facilement accessible », soit 90,1\% 《tout à fait d'accord » et 9,6 \% « plutôt d'accord » (Q. 10a).

En ce qui concerne les usages, la marche est de loin l'activité la plus pratiquée sur le site (Q. 3). À la question posée sur leur activité favorite sur le site, près de $90 \%$ (89,3\%) des personnes interviewées ont choisi cette réponse (la marche) parmi les choix de réponse offerts. Les autres activités préférées sont les suivantes : contempler la rivière $(45,9 \%)$, participer à différentes activités (danse, vélo, patins, jeux pour enfants, etc.) $(37,9 \%)$, faire des emplettes aux Halles ou au hangar $(39,8 \%)$ et rencontrer des gens ou des amis $(34,1 \%)$. Sur le même thème, les gens ont déclaré fréquenter régulièrement la promenade qui longe la rivière Saguenay ou les zones piétonnières (87,6\%). Le complexe des Halles et le secteur de l'étang et des jardins sont empruntés sur une base régulière par environ la moitié des répondants. La zone des jeux d'enfants n'attire que $19,5 \%$ des répondants qui prétendent visiter le site régulièrement. Cependant, cette zone semble utilisée assidûment par la clientèle des jeunes familles

\subsection{L'appropriation symbolique du site}

Le deuxième mode d'appropriation du site concerne les dimensions symboliques des pratiques des usagers. Trois questions portaient sur ce mode d'appropriation, soit Q. 10b, Q. 10e et Q. I0f. La question $\mathrm{IOb}$ renvoyait au registre de l'esthétique paysagère formelle. II s'agissait de savoir si les visiteurs se reconnaissaient dans le langage plastique retenu par les concepteurs. Selon les secteurs du site, celui-ci peut être associé au modèle pittoresque, qui mise sur des espaces où la végétation est très présente et structurée selon des formes souples et organiques, des matériaux tels que la pierre dans des formes brutes (bassin avec enrochement, ponceau et kiosque de bois, etc.). Dans d'autres secteurs de l'espace urbain, les aménagements s'inscrivent dans des référents plus contemporains, comportant des lignes plus formelles et davantage d'infrastructures et de matériaux « durs » ou minéralisés (fontaine de béton, granite, etc.). Toutefois, les questions ne distinguaient pas les secteurs, mais portaient plutôt sur l'ensemble du site.

Encore une fois, les usagers interrogés sont très satisfaits, puisque $98.5 \%$ disent être «tout à fait d'accord » ou "plutôt d'accord » avec l'affirmation selon laquelle «les aménagements paysagers sont réussis ॥ (voir Tableau 5). Deux autres questions concernaient l'appropriation symbolique du site, envisagée cette fois sous l'angle de l'expérience et du rapport à la nature. Les répondants affirment, pour la majorité d'entre eux, être en accord (tout à fait ou plutôt) avec l'idée que « le site permet de découvrir la rivière et le paysage $»(97,4 \%)$, tout comme avec celle que le « site permet le contact avec la nature » (93\%). Sur la base des réponses à ces trois interrogations, les choix proposés par les aménagistes semblent rejoindre la culture paysagère de la majorité des usagers interrogés sur la zone portuaire de Chicoutimi.

Par ailleurs, comme les données montraient des variations plus fortes selon l'âge et les revenus des répondants, nous avons voulu vérifier s'il y avait une relation significative entre ces deux variables du profil des répondants et ces trois indicateurs de l'appropriation symbolique du site. Les résultats des tests réalisés (Khi carré) sont concluants sous trois aspects. D'une part, il existe une relation significative entre l'âge du répondant et les deux affirmations «le site permet de découvrir la rivière et le paysage » $(\mathrm{P}=$ $0,006)$ et le « site permet le contact avec la nature » $(P=0,000)$. D'autre part, la relation est également significative, quoique moins forte, entre le revenu du répondant et l'affirmation «le site permet le contact avec la nature» $(P=0,023)$. 
EUE • L'appropriation des sites et paysages urbains requalifiés • b- I 3

Tableau 5

L'appropriation symbolique du site par les répondants

\begin{tabular}{|c|c|c|c|c|c|}
\hline & \multicolumn{2}{|c|}{ Âge } & \multicolumn{2}{|c|}{ Revenu } & \multirow[b]{2}{*}{ Total } \\
\hline IOb. Les aménagements paysagers & Moins de & 50 ans & Moins de & $\$ 50000$ & \\
\hline sont réussis? & 50 ans & et plus & $\$ 50000$ & et plus & \\
\hline Tout à fait d'accord & $78,7 \%$ & $84,1 \%$ & $82,4 \%$ & $79,3 \%$ & $81,6 \%$ \\
\hline Plutôt d'accord & $19,7 \%$ & $14,5 \%$ & $15,1 \%$ & $19,8 \%$ & $16,9 \%$ \\
\hline Plutôt en désaccord & $1,7 \%$ & $1,4 \%$ & $2,4 \%$ & $0,9 \%$ & $1,6 \%$ \\
\hline \multirow[t]{2}{*}{ Tout à fait en désaccord } & $0,0 \%$ & $0,0 \%$ & $0,0 \%$ & $0,0 \%$ & $0,0 \%$ \\
\hline & $\begin{array}{c}100 \% \\
(n=178)\end{array}$ & $\begin{array}{c}100 \% \\
(n=207)\end{array}$ & $\begin{array}{c}100 \% \\
(n=205)\end{array}$ & $\begin{array}{c}100 \% \\
(n=116)\end{array}$ & $100 \%$ \\
\hline I0e. Le site permet de découvrir & Moins de & 50 ans & Moins de & $\$ 50000$ & Total \\
\hline la rivière et le paysage? & 50 ans & et plus & $\$ 50000$ & et plus & \\
\hline Tout à fait d'accord & $73,6 \%$ & $85,0 \%$ & $85,4 \%$ & $72,4 \%$ & $79,7 \%$ \\
\hline Plutôt d'accord & $21,9 \%$ & $14,0 \%$ & $13,2 \%$ & $22,4 \%$ & $17,7 \%$ \\
\hline Plutôt en désaccord & $4,5 \%$ & $0,5 \%$ & $1,5 \%$ & $4,3 \%$ & $2,3 \%$ \\
\hline \multirow[t]{2}{*}{ Tout à fait en désaccord } & $0,0 \%$ & $0,5 \%$ & $0,0 \%$ & $0,9 \%$ & $0,3 \%$ \\
\hline & $\begin{array}{c}100 \% \\
(n=178)\end{array}$ & $\begin{array}{c}100 \% \\
(n=207)\end{array}$ & $\begin{array}{c}100 \% \\
(n=205)\end{array}$ & $\begin{array}{c}100 \% \\
(n=116)\end{array}$ & $100 \%$ \\
\hline I Of. C'est un espace vert qui permet & Moins de & 50 ans & Moins de & $\$ 50000$ & Total \\
\hline le contact avec la nature? & 50 ans & et plus & $\$ 50000$ & et plus & \\
\hline Tout à fait d'accord & $52,2 \%$ & $80,7 \%$ & $71,2 \%$ & $64,7 \%$ & $67,5 \%$ \\
\hline Plutôt d'accord & $35,4 \%$ & $16,9 \%$ & $22,4 \%$ & $27,6 \%$ & $25,5 \%$ \\
\hline Plutôt en désaccord & $12,4 \%$ & $2,4 \%$ & $6,3 \%$ & $7,8 \%$ & $7,0 \%$ \\
\hline \multirow[t]{2}{*}{ Tout à fait en désaccord } & $0,0 \%$ & $0,0 \%$ & $0,0 \%$ & $0,0 \%$ & $0,0 \%$ \\
\hline & $\begin{array}{c}100 \% \\
(n=178)\end{array}$ & $\begin{array}{c}100 \% \\
(n=207)\end{array}$ & $\begin{array}{c}100 \% \\
(n=205)\end{array}$ & $\begin{array}{c}100 \% \\
(n=116)\end{array}$ & $100 \%$ \\
\hline
\end{tabular}




\subsection{L'appropriation politique du site}

Le troisième axe d'analyse touche ce que nous appelons l'appropriation sociopolitique du site. Celle-ci est déclinée en deux ensembles de dimensions. D'entrée de jeu, disons que cet axe se distingue des deux autres, d'une part, parce qu'un taux plus élevé de personnes ont refusé de répondre (surtout des personnes plus jeunes ayant refusé de répondre à des questions concernant leur revenu), d'autre part, parce que les réponses obtenues sont moins consensuelles. Deux questions sont relatives à la maîtrise sociale du territoire (voir Tableau 6). Entre autres, il était demandé aux visiteurs de nous dire s'ils considéraient que «la zone portuaire a été aménagée en tenant compte des besoins de la population »(Q. I0j). Si la majorité des personnes interrogées répondent positivement, on observe néanmoins que leurs réponses sont réparties de manière plus équilibrée entre l'option «tout à fait d'accord» $(51,5 \%)$ et l'option "plutôt d'accord » (40,1 \%). Dans un deuxième temps, nous demandions aux usagers de se prononcer sur l'énoncé suivant : « Le site contribue à ma qualité de vie »(Q. IOh). À ce chapitre, il ressort que $93,1 \%$ des répondants sont d'accord avec cette affirmation, c'est-à-dire que $65,4 \%$ se disent « tout à fait d'accord » alors que $27,7 \%$ choisissent l'option de réponse « plutôt d'accord ».

Pour les deux questions, l'âge semble être un facteur qui influence la réponse. Pour la première question, $11,3 \%$ des répondants de moins de 50 ans sont soit «plutôt en désaccord » ou « tout à fait en désaccord » avec l'affirmation proposée, comparativement à $6 \%$ pour ceux ayant 50 ans et plus. Pour la seconde question, si l'on s'attarde uniquement au choix de réponse «tout à fait d'accord $», 48,7 \%$ des gens de moins de 50 ans ont choisi cette réponse, comparativement à $79,4 \%$ des gens de 50 ans et plus. Ce constat est soutenu par des tests de Khi carré qui indiquent des relations significatives: les personnes âgées de 50 ans et plus tendent à être plus positives sur ces deux aspects de l'aménagement, à savoir, s'il a été fait en fonction de leurs besoins et de la contribution du site à leur qualité de vie (les probabilités associées aux tests sont respectivement de 0,008 et de 0,000). On observe aussi, à la lumière des données recueillies, des différences entre les avis des répondants selon leurs revenus. II n'y a cependant pas de relations significatives selon les tests de Khi carré réalisés (respectivement 0,487 et 0,219 sur les deux questions).
Trois autres questions concernant l'appropriation politique touchent l'univers des rapports qui sont vécus avec les autres au sein de cet espace public (voir Tableau 7). Une de ces questions cherche à situer la vocation de cet espace public, qui pourrait créer ou contribuer à renforcer les liens sociaux des citadins, comme il est souvent postulé dans les théories et pratiques urbanistiques. On constate que pratiquement deux personnes interrogées sur trois $(65,9 \%)$ sont « tout à fait d'accord » avec l'affirmation que la zone portuaire "est un lieu de rencontre important à Chicoutimi », taux qui monte à $96 \%$ lorsqu'on ajoute les tenants de l'option "plutôt d'accord»(Q. 10c). Rappelons que, dans une autre question, le tiers affirmait venir personnellement sur la zone pour « rencontrer des gens ou des amis».

Deux interrogations sont en lien avec les thèmes de l'identité et de l'altérité à l'intérieur de cette grande catégorie conceptuelle de l'appropriation sociopolitique. La première se lit comme suit: " La zone portuaire représente bien l'image de Chicoutimi ॥ (Q. 10i). 93\% des individus interviewés sont d'accord avec cette affirmation $(62,5 \%$ - « tout à fait d'accord» et 30,7\% - "plutôt d'accord »). La deuxième interrogation se résume à l'énoncé « Je suis fier de présenter le site à des visiteurs de l'extérieur ॥ (Q. 10g). Les répondants acquiescent en ce sens dans une proportion de $98,4 \%(76,1 \%$ - «tout à fait d'accord" et 22,3\% - "plutôt d'accord»). Encore une fois, on observe une relation significative entre l'âge du répondant et ces trois indicateurs de l'appropriation politique du site: ceux plus âgés tendent à être plus positifs sur les trois questions (les probabilités associées aux tests de Khi carré sont respectivement de 0,000 , de 0,006 et de 0,000$)$. $\grave{A}$ l'inverse, il n'y a pas de relation significative mesurée entre le revenu et les réponses obtenues, même si les données montrent des variations. 
EUE • L’appropriation des sites et paysages urbains requalifiés • b- I 5

Tableau 6

L'appropriation politique par les répondants : volet «Maîtrise sociale du site»

\begin{tabular}{|c|c|c|c|c|c|}
\hline & \multicolumn{2}{|c|}{ Áge } & \multicolumn{2}{|c|}{ Revenu } & \multirow[b]{2}{*}{ Total } \\
\hline I0j. La zone a été aménagée en & Moins de & 50 ans & Moins de & $\$ 50000$ & \\
\hline fonction des besoins? & 50 ans & et plus & $\$ 50000$ & et plus & \\
\hline Tout à fait d'accord & $4 I, 3 \%$ & $59,9 \%$ & $53,6 \%$ & $53,8 \%$ & $5 \mathrm{I}, 5 \%$ \\
\hline Plutôt d'accord & $47,3 \%$ & $34,1 \%$ & $37,4 \%$ & $40,9 \%$ & $40,1 \%$ \\
\hline Plutôt en désaccord & $9,3 \%$ & $4,9 \%$ & $6,7 \%$ & $5,4 \%$ & $6,9 \%$ \\
\hline \multirow[t]{2}{*}{ Tout à fait en désaccord } & $2,0 \%$ & $1,1 \%$ & $2,2 \%$ & $0,0 \%$ & $1,5 \%$ \\
\hline & $\begin{array}{c}100 \% \\
(n=150)\end{array}$ & $\begin{array}{c}100 \% \\
(n=182)\end{array}$ & $\begin{array}{c}100 \% \\
(n=179)\end{array}$ & $\begin{array}{l}100 \% \\
(n=93)\end{array}$ & $100 \%$ \\
\hline IOh. Le site contribue à ma qualité & Moins de & 50 ans & Moins de & $\$ 50000$ & Total \\
\hline de vie comme citoyen? & 50 ans & et plus & $\$ 50000$ & et plus & \\
\hline Tout à fait d'accord & $48,7 \%$ & $79,4 \%$ & $70,3 \%$ & $60,6 \%$ & $65,4 \%$ \\
\hline Plutôt d'accord & $39,2 \%$ & $18,0 \%$ & $25,9 \%$ & $32,3 \%$ & $27,7 \%$ \\
\hline Plutôt en désaccord & $10,1 \%$ & $2,1 \%$ & $3,2 \%$ & $7,1 \%$ & $5,8 \%$ \\
\hline \multirow[t]{2}{*}{ Tout à fait en désaccord } & $1,9 \%$ & $0,5 \%$ & $0,5 \%$ & $0,0 \%$ & $1,2 \%$ \\
\hline & $\begin{array}{c}100 \% \\
(n=158)\end{array}$ & $\begin{array}{c}100 \% \\
(n=189)\end{array}$ & $\begin{array}{c}100 \% \\
(n=185)\end{array}$ & $\begin{array}{c}100 \% \\
(n=99)\end{array}$ & $100 \%$ \\
\hline
\end{tabular}


EUE • L'appropriation des sites et paysages urbains requalifiés • b- I 6

Tableau 7

L’appropriation sociopolitique par les répondants : volet «Rapport à l'altérité»

\begin{tabular}{|c|c|c|c|c|c|}
\hline \multirow[b]{2}{*}{ I0c. C'est un lieu de rencontre } & \multicolumn{2}{|c|}{ Áge } & \multicolumn{2}{|c|}{ Revenu } & \multirow[b]{2}{*}{ Total } \\
\hline & Moins de & 50 ans & Moins de & $\$ 50000$ & \\
\hline important à Chicoutimi? & 50 ans & et plus & $\$ 50000$ & et plus & \\
\hline Tout à fait d'accord & $45,9 \%$ & $82,5 \%$ & $69,8 \%$ & $60,8 \%$ & $65,9 \%$ \\
\hline Plutôt d'accord & $47,1 \%$ & $15,9 \%$ & $27,5 \%$ & $32,0 \%$ & $30,1 \%$ \\
\hline Plutôt en désaccord & $5,7 \%$ & $1,6 \%$ & $2,1 \%$ & $6,2 \%$ & $3,5 \%$ \\
\hline \multirow[t]{2}{*}{ Tout à fait en désaccord } & $1,3 \%$ & $0,0 \%$ & $0,5 \%$ & $1,0 \%$ & $0,6 \%$ \\
\hline & $\begin{array}{c}100 \% \\
(n=157)\end{array}$ & $\begin{array}{c}100 \% \\
(n=189)\end{array}$ & $\begin{array}{c}100 \% \\
(n=189)\end{array}$ & $\begin{array}{l}100 \% \\
(n=97)\end{array}$ & $100 \%$ \\
\hline I0i. La zone portuaire représente bien & Moins de & 50 ans & Moins de & $\$ 50000$ & Total \\
\hline I'image de Chicoutimi? & 50 ans & et plus & $\$ 50000$ & et plus & \\
\hline Tout à fait d'accord & $52,6 \%$ & $70,5 \%$ & $67,4 \%$ & $56,4 \%$ & $62,5 \%$ \\
\hline Plutôt d'accord & $39,1 \%$ & $23,8 \%$ & $27,8 \%$ & $33,7 \%$ & $30,7 \%$ \\
\hline Plutôt en désaccord & $7,1 \%$ & $4,1 \%$ & $3,2 \%$ & $7,9 \%$ & $5,4 \%$ \\
\hline \multirow[t]{2}{*}{ Tout à fait en désaccord } & $1,3 \%$ & $1,6 \%$ & $1,6 \%$ & $2,0 \%$ & $1,4 \%$ \\
\hline & $\begin{array}{c}100 \% \\
(n=156)\end{array}$ & $\begin{array}{c}100 \% \\
(n=193)\end{array}$ & $\begin{array}{c}100 \% \\
(n=187)\end{array}$ & $\begin{array}{c}100 \% \\
(n=101)\end{array}$ & \\
\hline l0g. Je suis fier de présenter le site & Moins de & 50 ans & Moins de & $\$ 50000$ & Total \\
\hline à des visiteurs de l'extérieur? & 50 ans & et plus & $\$ 50000$ & et plus & \\
\hline Tout à fait d'accord & $61,3 \%$ & $88,8 \%$ & $81,1 \%$ & $71,4 \%$ & $76,1 \%$ \\
\hline Plutôt d'accord & $35,7 \%$ & $10,7 \%$ & $17,9 \%$ & $26,7 \%$ & $22,3 \%$ \\
\hline Plutôt en désaccord & $2,4 \%$ & $0,5 \%$ & $0,5 \%$ & $1,9 \%$ & $1,4 \%$ \\
\hline \multirow[t]{2}{*}{ Tout à fait en désaccord } & $0,6 \%$ & $0,0 \%$ & $0,5 \%$ & $0,0 \%$ & $0,3 \%$ \\
\hline & $\begin{array}{c}100 \% \\
(n=168)\end{array}$ & $\begin{array}{c}100 \% \\
(n=196)\end{array}$ & $\begin{array}{c}100 \% \\
(n=196)\end{array}$ & $\begin{array}{c}100 \% \\
(n=105)\end{array}$ & $100 \%$ \\
\hline
\end{tabular}




\section{CONCLUSION}

Les grandes opérations d'aménagement visent à requalifier les espaces urbains, voire à leur donner un nouveau sens en termes de paysages et d'environnement urbains. Le substrat géoarchitectural serait issu de décisions qui s'additionnent au fil du temps comme résultat des mécanismes délibératifs. Les décisions touchant les grandes vocations d'un site sont généralement débattues dans des forums et lieux de discussion à caractère public. Ces décisions ont d'ailleurs retenu l'attention des chercheurs. Par contre, les décisions prises plus en aval, qui concernent différents aspects du programme d'activités, du design et de langage architectural, ont été moins étudiées. Pourtant, comme le relevaient des chercheurs intéressés à la problématique du paysage (Dubost et Lizet, 1995), des décalages culturels importants peuvent résulter de ces choix opérés en aval, la culture des professionnels n'étant pas forcément celle des groupes sociaux auxquels sont destinés les aménagements.

Notre hypothèse de départ était que le site de la zone portuaire de Chicoutimi avait adopté un concept d'aménagement en accord avec le discours « expert» en architecture du paysage. Ce style aurait pu affecter négativement le processus d'appropriation du site et du paysage par les usagers, même si le caractère public et «vert " du projet et ses grandes vocations correspondent aux demandes des intervenants sociocommunautaires ayant pris part aux débats des années 1980. Or, les résultats de notre sondage, viennent nuancer cette hypothèse.

D'une part, la zone portuaire de Chicoutimi se présente comme un paysage urbain requalifié qui est fortement apprécié par la très grande majorité des répondants, car il obtient de bons résultats dans les trois dimensions de concept d'appropriation, soit l'appropriation matérielle, l'appropriation symbolique et l'appropriation politique. Sur la base de ces résultats, le projet de réaménagement du «Vieuxport » de Chicoutimi s'affiche comme un succès du monde de l'architecture de paysage, de l'urbanisme et de l'aménagement du territoire. Fortement fréquenté, jugé « accessible», « réussi », « important» et «à notre image » par une forte majorité d'usagers interrogés, il participe à la qualité de vie de ces personnes, des citoyens provenant notamment des quartiers anciens avoisinants. II s'agit d'un espace public récréatif d'envergure, qui procure un lieu extérieur de rencontre dans une agglomération qui en compte relativement peu. Même s'il n'a pas permis une relance significative des activités commerciales et résidentielles de la zone centrale de l'arrondissement, cet espace contribue à l'urbanité d'un territoire marqué par les traditions rurales et par la vie quotidienne dans un environnement de type « banlieue pavillonnaire ». D'autre part, il importe de rappeler les limites de cette enquête. Celle-ci porte en effet sur les formes d'appropriation d'un groupe spécifique, c'est-àdire des visiteurs effectifs du parc urbain. Elle ne permet donc pas de comprendre le point de vue de citoyens qui ne le fréquentent pas, de façon délibérée ou non. En ce sens, notre enquête présente davantage la situation de fréquentation du site observée en 2008, qui est elle-même issue d'un processus de sélection qui s'est opéré sur une période de 17 ans, depuis l'ouverture du site au public.

Par ailleurs, dans ce portrait des usagers, quelques nuances semblent apparaître, des nuances qui pourraient ouvrir des pistes pour une investigation plus approfondie. Nous pensons notamment aux différences observées selon l'âge et les revenus des répondants. À partir d'analyses croisées, nous notons une relation significative entre le revenu (plus ou moins de $50000 \$$ par année) et la réponse pour une question seulement, alors que la relation est significative entre l'âge (plus ou moins de 50 ans) et les réponses pour sept des huit questions posées. En ce sens, les personnes âgées de 50 ans et plus sont généralement positives par rapport aux questions touchant l'appropriation symbolique et politique. Ainsi, certains profils d'usagers choisissent de fréquenter la zone portuaire requalifiée, alors que d'autres l'a délaissent.

Ce résultat d'enquête rejoint la question posée en introduction, à savoir « pour qui est conçu et aménagé ce type d'espaces publics? ». Le cas du «Vieux-port de Chicoutimi » semble démontrer que ces sites paysagés peuvent être très fréquentés et maintenir un aspect populaire. Pour l'avenir, il demeure pertinent de s'enquérir des mécanismes généraux déterminant la sélectivité du processus d'appropriation. Ce processus serait-il lié aux messages véhiculés par un concept matérialisé ou à la répétitivité des fréquentations par un groupe spécifique? Observerait-on un effet de voisinage ou de proximité comme l'a remarqué Arrif (2009) pour le parc de Bercy à Paris. Par ailleurs, plusieurs habitations pour personnes âgées sont localisées au centre-ville de l'arrondissement de Chicoutimi. L'âge est un facteur significatif pour les représentations spatiales. De plus, il peut être associé à d'autres variables importantes concernant le profil de l'usager, comme l'occupation, le temps disponible pour fréquenter le lieu, etc. Ces autres variables sont peut-être encore plus déterminantes pour les formes d'appropriation du parc urbain. II s'agit là de questions 


\section{EUE • L'appropriation des sites et paysages urbains requalifiés • b- I 8}

et d'hypothèses qu'il serait pertinent de considérer avec attention dans des analyses futures plus sophistiquées, comme des modèles de régression multiples ou de régression logistique ordonnée.

En rétrospective, les grands sites urbains sont l'objet de convoitise, dans les métropoles comme dans les villes moyennes. Différents groupes souhaitent y matérialiser leurs visions du territoire ainsi que les usages et formes qu'ils estiment garants du succès. À l'intérieur de ce contexte, les sites portuaires affichent des particularités. Espaces publics généralement situés au cœur des agglomérations urbaines, ils constituent des territoires clefs, tant du point de vue des représentations et symboles d'une communauté qu'en ce qui concerne l'implantation d'équipements collectifs. Ils participent également à une forme de reconquête du centre, notamment au sein des villes nord-américaines, qui se révèlent fortement touchées par le phénomène d'étalement urbain. II s'agit donc à la fois de lieux stratégiques sur les plans économique et fonctionnel et d'espaces forts, voire de hauts-lieux, dont le sens s'est construit socialement au fil du temps. II paraît donc compréhensible que ces territoires soient l'objet de tant de désirs, de projets, de débats et d'appropriations.

\section{BIBLIOGRAPHIE}

ARRIF, T. (2009) « Les représentations sociales liées au lieu de résidence au sein du parc de Bercy », Métropoles, no. 5, [En ligne], mis en ligne le 8 avril 2009. URL : http://metropoles.revues.org/document3876.html . Consulté le 26 avril 2009.

Augustin, J.-P. (2000). «Constructions de sites, aménagement et mémoires collectives: Trouver sa place, de place en place », in AUGUSTIN, J.-P. et SORBETS, C. (dir.) Sites publics, lieux communs. Aperçus sur l'aménagement de places et de parcs au Québec, Bordeaux, Maison des sciences de l'homme de l'Aquitaine, p. 9-34.

Gauthier, M.-J. et M. Simard (2009). Atlas électronique du Saguenay-Lac-Saint-Jean [En http://atlas.uqac.ca/saguenay-lac-saint-jean/index.php

BoIVIN, N. (1993). « Chicoutimi et le Vieux-port remportent trois prix », Progrès-Dimanche, 5 septembre, p. 4.

BONIN, S. (2008) «Fleuves en ville: enjeux écologiques et projets urbains », Strates [En ligne], Numéro 13-2007 Paysage urbain: genèse, représentations, enjeux contemporains, Mis en ligne le : 22 octobre 2008, Disponible sur http://strates.revues.org/document5963.html, Consulté le 12 décembre 2008.
CADIOU, N. et Y. LUGINBÜHL (1995). « Modèles paysagers et représentations du paysage en Normandie-Maine», Paysage au pluriel. Pour une approche ethnologique des paysages. Coll. Ethnologie de la France, cahier $n^{\circ} 9$ : 19 34.

CONSEIL DE L'Europe (2000). Convention européenne du paysage, STCE no. 176.

CÔTÉ, D. (1993a). «Animation de la zone portuaire: le conseil municipal de Chicoutimi approuve la création d'une corportion », Progrès-Dimanche, 23 février, p. 13.

CÔTÉ, D. (1993b). «Le Vieux-port épargné par les vandales », Progrès-Dimanche, I août, p. 8.

COURCIER, S. (2008). « L'effet structurant d'un projet urbain : le cas du Vieux-Port de Montréal », in Gauthier, M, M. GARIÉPY et M.-O. TRÉPANIER (dir.) (2008). Renouveler l'aménagement et l'urbanisme. Planification territoriale, débat public et développement durable, Montréal, Presses de l'Université de Montréal, 329 pages.

Davies, W.K.D. et D.T. Herbert (1993). Communities Within Cities. An Urban Social Geography, Belhaven Press, London (U.K.), 256 pages.

Dubost, F. et B. LIZET (1995). «Pour une ethnologie du paysage », Paysage au pluriel. Pour une approche ethnologique des paysages. Coll. Ethnologie de la France. Cahier $n^{\circ} 9: 225-240$.

EPSTEIN, J.-A. ( 1981 ). « Les paysages : espaces sans nom», Les annales de la recherche urbaine, Nos I0-I I : 54-8I.

FORTIN, M.-J. (2005). Paysage industriel: lieu de médiation sociale et enjeu de développement durable et de justice environnementale. Les cas des complexes d'Alcan (Alma, Québec) et de Pechiney (Dunkerque, France), Thèse de doctorat présentée à l'Université du Québec à Chicoutimi et à l'Université Paris I Sorbonne (Accessible en ligne : http://theses.uqac.ca/these_24605668.html, consulté le 28 mars 2008).

Frémont, A. (1976). La région, espace vécu, Paris, Presses Universitaires de France, coll. SUP. 223 pages.

GuAY, J.-H. (199I). Sciences humaines et méthodes quantitatives : les principes d'application de la recherche, Laval, Beauchemin, 4I 2 pages.

Hossard, N. et M. Jarvin (dir.) (2005). C'est ma ville! De l'appropriation et du détournement de l'espace public, Paris, L'Harmattan, 285 pages.

JACOBS, J. (196I). The Death and Life of Great American Cities, New York, Vintage Books, 458 pages.

Larochelle, P. et D. Piché (1990). «L'aménagement du Zone portuaire de Québec: Question d'images et questions de recherche », dans MORIN, R., R. PETRELLI, D. Pilette et L.-N. Tellier (dir.) Gestion locale et problématiques urbaines au tournant des années 1990, Montréal, UQAM, p. 172-194.

LYNCH, K. (1989). Good City Form, Cambridge, Mass., MIT Press, 514 pages. 
MARSAN, J.-C. (199I). «L'aménagement du Zone portuaire de Montréal. Les avatars de l'urbanisme promoteur », dans Germain, A. (dir.) L'aménagement urbain : Promesses et défis, Québec, IQRC, p. 27-60.

Mumford, L. (198I). The Culture of Cities, Westport, Conn., Greenwood Press, 586 pages.

Pradel, B. (2007). « Mettre en scène et mettre en intrigue : un urbanisme festif des espaces publics », Géocarrefour, Vol. 82, no. 3 : I23-130.

RAFFESTIN, C. (1977). « Paysage et territorialité », Cahiers de géographie de Québec, Vol. 21 , nos. 53-54 : 123-134.

Semmoud, N. (2007). La réception sociale de l'urbanisme, Paris, L'Harmattan, $25 \mathrm{I}$ pages.

Serfaty-Garzon, P. (2003). «L'appropriation » dans SEGAUD M, BRUN J et DRIANT J-C (dir.) Dictionnaire critique de l'habitat et du logement, Paris, Éditions Armand Colin, p. 27-30.

SIMARD, M. (2000). «Développement local et identité communautaire : l'exemple du quartier Saint-Roch à Québec », Cahiers de Géographie du Québec, vol. 45, no. 122 : 167-188.

Simard, M. et MALTAIS, N. (2004). « Le croissant culturel et touristique de Chicoutimi. Une démarche de revitalisation par le biais des arts et de la culture ", Organisations et territoires, vol. 13, no. 2 : 93-101.

TREMBLAY, C. (1993). «L'achalandage [au Vieux-port] comble tous les espoirs », Progrès-Dimanche, 4 juillet, p.8.

TREMBLAY, S. (2004). À la recherche d'un autre développement: la dévitalisation urbaine et la revitalisation communautaire au centre urbain de Chicoutimi de 1960 à nos jours, Thèse de doctorat non-publiée, Université du Québec à Chicoutimi, Saguenay, 543 pages.

Villeneuve, P., C. Trudelle et M. Pelletier (2005). «L'accueil des croisières internationales au Québec: s'agit-il d'un projet de développement territorial? », dans Bhérer, L., J.-P. Collin, É. Kerrouche et J. Palard, (dir.) Jeux d'échelle et transformation de l'État: le gouvernement des territoires au Québec et en France, Québec, PUL, p. 257-288.

ZukIN S. (1995). The Cultures of Cities, Oxford, Blackwell, 313 pages. 